Voix et Images

volxetimages

\title{
Hubert Aquin : entre le littéraire et le théologal
}

\section{Josiane Leralu}

Volume 11, numéro 3 (33), printemps 1986

Yolande Villemaire

URI : https://id.erudit.org/iderudit/200583ar

DOI : https://doi.org/10.7202/200583ar

Aller au sommaire du numéro

\section{Éditeur(s)}

Université du Québec à Montréal

\section{ISSN}

0318-9201 (imprimé)

1705-933X (numérique)

Découvrir la revue

\section{Citer cet article}

Leralu, J. (1986). Hubert Aquin : entre le littéraire et le théologal. Voix et Images, 11(3), 495-506. https://doi.org/10.7202/200583ar d'utilisation que vous pouvez consulter en ligne.

https://apropos.erudit.org/fr/usagers/politique-dutilisation/ 


\title{
Hubert Aquin: entre le littéraire et le théologal
}

\author{
par Josiane Leralu, Université Mc Gill
}

En 1959, Hubert Aquin publie son premier et unique "récit»: les Rédempteurs. En 1981, la revue Liberté publie son dernier roman inachevé: Obombre. Deux titres particulièrement significatifs puisqu'ils appartiennent au vocabulaire théologique et marquent le début et la fin - choisis - de sa carrière littéraire. Dans Obombre, l'auteur avoue d'emblée: Derrière les artifices de l'intrigue (...), se cache une pauvre loque qui se prend pour Dieu. On peut donc se demander si l'entreprise romanesque - à laquelle notre étude se limite ${ }^{1}$ - ne s'affirme pas, de plus en plus, comme une entreprise théologale. Deux domaines référentiels constants et d'une importance majeure chez Aquin sont d'ailleurs très signifiants sur ce sujet: le théâtre et le religieux. On peut en voir les principales manifestations dans le comment dire et dans le quoi dire ${ }^{2}$.

\section{Comment dire}

\section{Le théâtre}

Toute l'œuvre romanesque d'Hubert Aquin exprime la nostalgie du théâtre. Rien d'étonnant à cela puisqu'il fit ses débuts littéraires dans le téléthéâtre, qu'il continua dans ce genre jusqu'en 1974 et que toutes ses pièces, ou presque, restèrent inédites de son vivant. Lui-même ne peut que constater cette obsession sans pouvoir l'expliquer ${ }^{3}$.

Le récit les Rédempteurs comporte déjà des éléments dramaturgiques, tels que la réduction du spatio-temporel et l'unicité de l'action. Par ailleurs, le récit débute au point culminant de la crise et le dénouement intervient en même temps que la résolution de cette crise. Enfin, les dialogues ont la part belle et il serait relativement aisé de réduire les passages narratifs à des remarques scéniques. Ce texte, pièce déguisée d'inspiration classique, ne peut manquer de nous renvoyer à la tragédie racinienne où les héros sont enclos dans l'univers qui aboutit à la mort. Ici, la mort sera métaphorique ou non: suicide collectif ou perpétuation de la mort à travers l'amour.

Dans Trou de mémoire, l'évocation théâtrale s'affirme comme une volonté consciente et organisée: la présentation typographique procède d'une perspective scénique: ....en haut de la page une arrière-scène, ensuite un pro-scénium, ensuite une avant-scène, puis une contre avant-scène qui correspond aux notes en bas de page (Q.L.2, 136). 
L'Antiphonaire amplifiera ce jeu par les transferts de personnages, d'époques, de lieux, d'œuvres, etc. Mais c'est dans Neige noire qu'il n'y aura plus seulement théâtralité mais bien théâtre. L'intégration d'artifices, d'un déroulement propre au théâtre, tout le côté spectaculaire - qui culminera dans Prochain épisode - s'affirme désormais et n'hésite plus à se nommer. Neige noire comporte trois niveaux d'écriture: celui du roman, celui de la pièce de théâtre et celui du scénario qui retranscrit la réalité et envahit peu à peu le roman qui s'amenuise en proportion. Le roman raconte, au fond, la répétition d'une même scène d'amour cristallisée dans le meurtre de Sylvie, expression tragique de l'impossible amour absolu entre deux êtres. Le roman se nourrit de théâtre (héros-comédien, références nombreuses à Hamlet par répétitions, identifications, superpositions) et de spectacle en général (scénario, observations devant un miroir, intégration d'émissions télévisées, rêves...) et se meurt par le théâtre qui l'aspire: Nicolas est "condamné» à renouveler son crime sur des pseudo-Sylvie. Théâtre d'autant plus tragique qu'il est voué au reflet donc à l'illusion de l'Absolu qui le motive.

De plus, toute une thématique des masques scéniques traverse l'œuvre d'Aquin: les personnages sont interchangeables (P.E.: poursuivant/poursuivi; N.N.: Sylvie/Eva/Linda) ainsi que les "fabricants" du livre, euxmêmes personnages (T. M.: narrateur/correcteur/éditeur/lecteur). À cela n'existe aucun obstacle d'espace ni de temps (A: Amérique du Nord au XX $\mathrm{X}^{\mathrm{e}}$ siècle / Europe de la Renaissance). Procédé d'autant plus aisé que les descriptions de personnages sont très succinctes et parfois même inexistantes. Mieux même: il se produit au long de l'œuvre aquinienne un transfert de narrateur-personnage à narratrice-personnage qui débouche finalement dans Neige noire sur un narrateur-type tel qu'exigé par l'écriture d'un scénario. Comme l'acteur grec par le jeu des masques, l'auteur se met en scène à travers différents rôles par les masques de l'écriture: homme/femme, meurtrier/victime, personnage/narrateur/correcteur/éditeur/lecteur à différents instants, en divers lieux. Cette fusion de l'Autre dans l'Un trahit une volonté d'être tout et partout à la fois et de repenser le monde, autrement dit d'être Dieu.

Le théâtre perspectiviste d'Aquin procède ainsi d'une réalité indéfiniment décomposée et recréée en vertu d'un Absolu fictionnel proposé comme réalité unique, vraie, totale et moteur de la création. Le pouvoir globalisant des arts visuels tend alors à résoudre une dialectique: unifier les multiples fragmentations de cette réalité, tout en restituant, comme dans la peinture cubiste, l'instantanéité et la multiplicité de la vie. Tout se passe comme si le projet romanesque d'Aquin était de discerner puis de reproduire une multiplicité maximale d'aspects - dans le temps et dans l'espace - et de les orchestrer pour en faire une œuvre irremplaçable et, de préférence, l'Oeuvre ${ }^{4}$. Ainsi, on ne peut s'étonner de retrouver des références de plus en plus amplifiées à la Bible, récit par excellence. 


\section{Le religieux}

On ne peut aborder cette question sans penser d'abord à l'anticléricalisme avoué d'Aquin. En définissant ce que. l'on refuse, on suggère aussi ce que l'on souhaite. Cette préoccupation anticléricale d'Aquin traduit, par opposition, une préoccupation religieuse.

La révolte d'Aquin se manifeste essentiellement dans le vocabulaire: il utilise à profusion les jurons, en crée d'autres et les fait participer totalement au discours: Je bascule dans le surblasphème avec la ferveur des premiers apôtres (T. M., 94). Comme on le sait, dans la culture québécoise, ce lexique emprunté au domaine eschatologique ou liturgique se trouve entièrement catalogué dans le langage parlé. Or, avant Aquin, les auteurs n'endossaient pas la responsabilité du langage blasphématoire de leurs personnages et revendiquaient encore moins ce langage dans leur propre discours.

Ce langage intègre et dénonce à la fois un certain contexte sociohistorique québécois. Il menace aussi ce qui le fait exister: le langage littéraire. La partie (jurons) lutte contre le tout (langage). Il n'y a plus métonymie ni même métaphore mais éclatement de ces images à l'intérieur et au moyen de ce qui les fait exister: la littérature. Ainsi, ce choix langagier se manifeste comme une autre forme de l'acte transgressif instaurateur de la tragédie qui se retrouve tout au long de l'œuvre romanesque d'Aquin et qui prend la forme du suicide, du meurtre ou du viol, que l'auteur définissait par ces mots: On pourrait (...) ramener cela à quelque chose comme le meurtre fondamental. C'est l'acte transgressif instaurateur de la tragédie (Q.L.2, 136).

Mais, s'il y a volonté d'opposition, qui culmine dans Trou de mémoire. il y a aussi séduction. Là encore, le vocabulaire est signifiant. Il suffit de relever les titres: la connotation liturgique est présente dans l'Antiphonaire et la référence biblique dans les Rédempteurs et Obombre ${ }^{5}$. Neige noire et Point de fuite expriment une volonté de résoudre les contraires en une seule entité. Et n'est-ce pas là une des définitions de Dieu? La séduction se manifeste ici par le désir de se substituer à celui qui séduit. Or, ce projet se trouvait déjà inscrit dans les Rédempteurs où les hommes sont présentés comme l'élément qui dégrade la création. Par leur suicide-sacrifice collectif, ils projettent de sauver le monde en lui rendant sa perfection perdue. En se sacrifiant pour rediviniser le monde, ils se transforment chacun en Christ rédempteur. De même, Christine, la narratrice au prénom signifiant de l'Antiphonaire, est à la fois officiante et victime; et son suicide sacrificiel est voué à un éventuel progrès de la vie présente.

Comme Christine, les autres personnages du roman sont successivement immolés. Survivent Robert et son épouse Suzanne qui perpétueront le mal de vivre par une étreinte (qui) peuplera le monde (R., 113).

L'œuvre romanesque d'Aquin est ainsi faite qu'en voulant résister au théologal, moteur de son désir de création littéraire, il résiste aussi à la littérature et à l'Oeuvre. D'où ce recommencement perpétuel et cette insatis- 
faction ininterrompue. Cette dialectique se manifeste tout naturellement dans le sacrilège à la fois verbal et structurel. Comme l'a montré Patricia Smart, Trou de mémoire, en opposition au tableau de Holbein, représente un immense blasphème ${ }^{6}$. Mais c'est dans l'Antiphonaire que ce défi sacrilège devient parfaitement explicite et autonome. Le roman reproduit le modèle du chant antiphonique et les larges extraits du Cantique des Cantiques provoquent l'extase physique mise en parallèle avec la transe mystique.

À la suite de Gilles de la Fontaine, on peut voir l'ironie qui se dégage de ce roman, messe parodique: Christine/Christ, fidèles/infidèles, etc. Et on ne peut non plus ignorer les propres commentaires d'Hubert Aquin qui expliquait: la parenté qu'il y a entre les textes érotiques intenses et les textes mystiques (Q.L.2, 137). La polysémie du texte est pleinement agissante chez Aquin et Neige noire vient confirmer notre hypothèse: ce roman est clos en apothéose, dans tous les sens du terme: il y a déification (Le Christ s'est réincarné en toi, 251) et sublimation de l'amour (Voici donc venu le véritable amour, 252). Il est aussi intéressant de noter que le Verbe est entré (N.N., 253) en Eva et que le choix de ce prénom ne peut être innocent chez Aquin: la femme est perdition et salut (palingénésie, 253) ${ }^{7}$. Le couple Renata-abbé Chigi se reforme dans le couple Eva-Linda. L'extase physique parle de l'extase mystique qui est elle-même fusion des contraires: Sa chevelure blonde descend dans les cheveux bruns d'Eva, 251.

Or, si dans une analyse intertextuelle on ne tient pas compte de l'entreprise littéraire d'Aquin comme une entreprise théologale, les quatre dernières pages du roman, qui sont absolument essentielles, ne peuvent qu'apparaître plaquées. Aquin lui-même affirmait qu'il n'y avait pas là distanciation mais bien drame: ...obsession, à tel point que j'ai abandonné tout procédé d'humour et d'ironie dans Neige Noire. Je me suis retrouvé en présence de quelque chose qui me dramatisait de l'intérieur (Q.L.2, 135). Le sacrifice qui pouvait sembler parodie au départ devient tragédie.

Ainsi, la séduction théologale, latente dans les Rédempteurs, puis amorcée dans Prochain épisode, est structurellement refusée dans Trou de mémoire. Elle devient plus explicite et autonome dans l'Antiphonaire pour enfin s'exprimer lucidement dans Neige noire. Et dans Obombre, plus aucun artifice ne réussit à voiler le projet: dès la première phrase, l'auteur lance un cri éperdu.

Chemin faisant, l'œuvre d'Aquin révèle donc un projet qui la déborde et qui se dépouille peu à peu de ses artifices mystificateurs (esthétique baroque), de ses fausses pistes (roman policier original) ou de ses intentions plus explicites (révolution littéraire/ révolution politico-sociale).

Avant d'aller plus loin, nous laisserons la parole à Aquin-narrateur dans Neige noire puisque ce roman exprime, pensons-nous, la synthèse de sa démarche romanesque et théâtrale en même temps que l'évidence de son entreprise théologale: 
Le temps me dévore, mais de sa bouche, je tire mes histoires, de sa sédimentation mystérieuse, je tire ma semence d'éternité. Eva et Linda approchent de ce théâtre illuminé où la pièce qu'on représente est une parabole ${ }^{8}$ dans laquelle toutes les ceuvres humaines sont enchâssées, 254.

Ainsi, le théâtre et le religieux s'unissent pour produire l'œuvre romanesque et parler d'elle.

Cependant, il ne suffit pas d'envisager la parabole sous son aspect formel. Encore faut-il que le quoi dire s'intègre à l'allégorie pour la rendre valide. Nous chercherons donc à dégager le message auquel la parabole veut donner tout son sens.

\section{Quoi dire}

Puisque la parabole est utilisée pour rendre le sacré sensible à un auditoire, la relation lecteur/narrateur ainsi que le thème du mythe deviennent essentiels.

\section{La relation lecteur/narrateur}

Dans Prochain épisode, lecteur et narrateur se cherchent, ce qui produit le jeu incessant des chassés-croisés (narrateur/H. de Heutz/K.). La rencontre a lieu dans Trou de mémoire où le narrateur tour à tour séduit, déroute, agresse le lecteur qui devient alors le personnage central vers lequel tous les effets sont dirigés, pour s'en désintéresser ensuite. l'Antiphonaire composé en forme d'aura épileptique souligne à quel point le scripteur est seul. Tout le côté spectaculaire est désormais introverti. Il y a dissolution de la conscience envahie par "l'outre-conscience»:

...l'épilepsie est quelque chose d'infiniment solitaire et dévorant qui isole de tout quand cela arrive et, quand cela n'arrive pas, cela est redouté, et quand c'est arrivé on s'en souvient.

Ainsi, l'épileptique est entièrement centré sur son mal sacré, facilement assimilable au mal d'écrire. Le lecteur est donc supprimé. Il faut dire aussi que dans Trou de mémoire, le lecteur n'existe pas pour lui-même. En fait, le lecteur-éditeur est le double complémentaire de l'auteur-éditeur.

Même dans ce roman où le lecteur semble avoir son rôle à jouer, Aquin répète-t-il l'identité: il se regarde, s'aime, se prolonge, se multiplie ou se déteste dans son propre double. De sorte que le lecteur se voit confiné dans un rôle de voyeur auquel on refuse toute participation à la création littéraire. Mais il peut, à la rigueur, assister à son avènement. Ce voyeurisme, présent déjà dans Prochain épisode (à partir notamment de la description complaisante de scènes érotiques) est développé comme principe esthétique dans Neige noire. Selon Aquin le lecteur/spectateur/auditeur devrait aborder l'cuvre, masqué (N.N. 159). Ce qui revient à ne plus accepter que le regard et à refuser au lecteur toute personnalisation. Le lecteur métaphorisé devient partie intégrante de l'œuvre. Là encore, le scripteur se veut partout à la fois, 
prévoyant tout (réactions du lecteur comprises), concevant tout, orchestrant tout : c'est en effet le grand compositeur omniprésent, omnipuissant et qui se proclame tel.

Or, en refusant tout imprévu, le scripteur refuse du même coup l'instantanéité régénératrice de la lecture. Le lecteur, réduit au rôle de voyeur, ne peut qu'occuper la place qui lui a été assignée. Et le scripteur se retrouve seul avec sa création voulue comme un tout prévu et quantifié totalement. Une fois de plus, nous retrouvons plusieurs similitudes avec le schéma théologal.

Cette volonté d'auto-création perpétuelle, vivante, aboutit à une instabilité stylistique. Et paradoxalement, cette agitation confine à l'immobilisme car elle ne dépasse pas une remise en question continuelle, répétitive. Au lieu d'engendrer la vie - donc la création incessante - l'auteur engendre un enfermement de l'œuvre dans sa propre contemplation. Il n'existe dès lors aucun mouvement générateur mais simplement une répétition surmultipliée d'une même image à l'aide de multiples miroirs réfléchissants. Or, la création divine est à la fois achevée (définitive, prévue, quantifiée) et en constante évolution (l'homme et la nature tout entière participent à la création). Aquin n'a pu résoudre cette dichotomie. Tout est calculé, trop calculé et sans le lecteur. Aquin a beau parler du lecteur comme co-créateur de l'œuvre9, en réalité, l'œuvre est construite sans lui. Tel Nicolas, qui dans Neige noire répète son crime, Aquin doit se contenter du semblable quand il voudrait reproduire l'identique. Ainsi, Aquin et son cuvre "meurent-ils" de cette impossibilité ontologique involontaire et inévitable qui les condamne au leurre du théâtre illusionniste, de l'esthétique baroque, de la fiction, des miroirs réfléchissants qui transforment l'image initiale en un de ses reflets. L'œuvre et le narrateur sont condamnés à vivre dans le Double, dans l'Ombre, alors qu'ils cherchaient ce chemin de lumière et d'euphorie (P. E., 35).

L'œuvre se donnant comme parfaitement déterminée, tout se passe comme si le dialogue finissait par se situer bien au-delà de la relation lecteurauteur. Le dialogue véritable s'instaure entre l'écrivain et son œuvre qui le transcende et se transcende elle-même. L'auteur-dieu s'installe dans une solitude infinie (N.N., 149), contemplant sa création, et le lecteur, intégré à cette création, se trouve aussi absorbé par elle, sans réel pouvoir sur elle, prisonnier de celle-ci (N.N., 158).

Cependant, puisque l'œuvre dépend toute de l'auteur-homme (avant d'être l'auteur-dieu), cette création ne peut être qu'épuisante: en même temps qu'elle manifeste l'existence du créateur, elle se nourrit de lui seul, ne lui laissant aucun répit, contrairement à la création divine qui, elle, est conçue sans effort (cf. Dieu dit et cela fut). Dans une relation lecteur-auteur qui place le lecteur à l'extérieur de la création, l'auteur partage la création avec le lecteur et peut se reposer sur la lecture créatrice et le fortuit. Ce qui n'est pas le cas chez Aquin dont les livres sont basés sur le principe de l'auto-analyse et de l'explication. Ainsi, Aquin explique et "démonte» le tableau de Holbein au lieu de s'en remettre à la culture du lecteur. Ce constant parti-pris d'a-culture du lecteur s'exprime souvent sous la forme d'une désinvolture ironique (cf. toutes les références bio - et bibliographiques en notes). 
Le moi, à force de prendre ses distances avec la réalité, finit par échapper à son emprise, s'isole et reconstitue sa propre réalité par un dédoublement en plusieurs «JE»: narrateur/éditeur/lecteur/hommes/femmes/etc.: le roman c'est moi (T. M., 19). L'auteur se transmue littéralement dans son œuvre et plus l'œuvre grandit, plus Aquin diminue telle une peau de chagrin. Dans ces conditions, on comprend fort bien que l'auteur ait pu dire à sa femme, avant de se suicider: Je n'ai plus aucune réserve en moi. Je me sens détruit ${ }^{10}$.

Compte tenu de l'attrait prôné par Aquin pour la littérature nordique ou d'inspiration nordique, on pense à la mythologie nordique selon laquelle:

L'héroisme est le seul soutien possible, le seul bien pur et sans tache donné à l'esprit humain et l'héroïsme se fonde sur des causes perdues. Ce n'est donc qu'en mourant que le héros peut donner la preuve de sa valeur ${ }^{11}$.

Chez Aquin, la similitude entre la condition de l'écrivain et la destinée du héros nordique est frappante: l'acte d'écrire, assimilé à une entreprise théologale, est acte héroïque. Il sort l'homme de sa condition, le hausse au rang de héros (i.e. demi-dieu), le maintient en vie jusqu'au jour fatal où il lui faudra accomplir le geste qui donnera tout son sens à l'entreprise littéraire: Écrire me tue (P.F., 48). Ainsi, on ne peut dissocier l'acte d'écrire du mythe.

\section{Le mythe, le sacré, le profane}

Écrire traduit chez Aquin l'obsession de la "grandeur perdue", point de départ du récit les Rédempteurs. Après plus de dix ans de "silence romanesque", il reprend ce mythe de la Genèse sous une forme voilée. Il écrivait d'ailleurs, en 1952:

Je crains de ne jamais pouvoir écrire quoi que ce soit qui ne reprenne fatalement Les Rédempteurs. Prisonnier de ma propre histoire, cela me parait inévitable (P.F., 48).

Tout ce qui précède a déjà montré la répétition intertextuelle: le mythe de la faute originelle qui interdit l'accès à l'absolu imprègne toute son œuvre. Or, si le sacré disparaît, le mythe tragique éclate: il n'y a plus alors ni faute, ni tragédie puisque la transgression naît de l'existence du sacré. Aquin tente donc de désacraliser (blasphèmes, médicaments, notes démystificatrices, anticléricalisme...) et pourtant resacralise: sacré, je le suis (T. M., 21). Aussi écrit-il au maximum de la fureur et de l'incantation (T. M., 35). Cette dialectique se résoud dans le défi à Dieu qui est simultanément résistance explicite (veine sacrilège) et reconnaissance implicite (resacralisation).

Profaner, ce n'est pas seulement vouloir abolir le sacré c'est aussi chercher à briser les barrières qui retiennent l'homme en-deça de la plénitude: c'est toute la thématique du viol qui traverse l'œuvre romanesque d'Aquin.

À partir de là s'inscrivent toutes les déviations sexuelles, métamorphoses de la transgression par excellence qui procure la sensation de plénitude extatique. Ainsi, Olympe Ghezzo-Quenum, identifié par R.R. à son violeur 
P.X. Magnant explique: Unis dans cette étreinte (...) nous avons continué cette course exaltée vers un terme, hélas, trop rapproché (T. M., 183). Seule, la transgression engendre l'extase: la relation conjugale qui lie Sylvie et Nicolas est placée sous le signe de l'impuissance qui n'existait pas avant le mariage. Mais, nécessairement, puisqu'il y a "faute", le plaisir, pour intense qu'il soit, n'est ni pur (moi, désespéré, T. M., 183) ni entier (elle, inconsciente, T. M., 183). Le plus souvent même, cette plénitude ne peut se savourer qu'entachée de sacrilège (conséquence de la faute initiale profanatrice): on pense au viol de Renata par l'abbé Chigi mais aussi à l'adultère et au sadomasochisme avec Linda, à l'inceste Sylvie/Michel ou au lesbianisme Eva/ Linda (qui, lui, sera transcendé par l'amour divin et de ce fait, pourra se muer en extase durable, N.N., 252).

Ce mythe de la déchéance de l'homme devenu incapable de vivre un amour absolu sans aide divine s'apparente au projet scripturaire qui parle de l'objet impossible. Si l'Amour absolu pouvait s'inscrire dans la durée, à l'échelle humaine, l'homme éclipserait Dieu. Écrire ne serait plus alors un pâle reflet de l'Oeuvre inimitable (à force de paraphraser l'innommable..., P. E., 22).

Écrire, c'est donc tenter de susciter, maîtriser et servir simultanément un sacré qui peut résister (d'où prise de médicaments) ou surgir sans avoir été appelé (épilepsie $=$ "mal sacré») ou avoir été profané (les noms sont sacrés dans Neige noire). Et c'est justement cette impossibilité de retenir le sacré (fuite incessante dans Prochain épisode ou apparition perturbatrice dans l'Antiphonaire) que va éprouver Aquin sans pouvoir l'expliquer.

L'écriture et le sacré ont cela en commun qu'ils ne s'expliquent qu'en existant et que, paradoxalement, leur existence manifeste une résistance à toute réduction. Écrire, c'est donc tuer: tuer le Récit en cherchant à le matérialiser. À ce titre, nous percevons les meurtres et suicides qui abondent dans l'cuvre d'Aquin comme des métaphores de l'acte d'écrire. Mais, peu à peu, l'immolation systématique des personnages ne suffit plus. L'œuvre, entreprise avec la conviction que le mot tue (P. E., 164), s'achemine vers la particularisation: écrire me tue (P. E., 48). L'écriture d'Aquin était suicidaire dès le départ et il le savait (P.E., 25-26). D'ailleurs, tous les titres de ses romans traduisent ce qui est absence, impossibilité (du livre inédit). La mort, réalité vécue à travers l'écriture, fait de l'ouvre une MESSE SACRIFICIELLE. On comprend alors que l'écrit s'apparente au sacré et soit, successivement ou simultanément, incantation et répulsion car, dans cette perspective, seule la mort est pleinement textuelle.

De cette manière, tout un monde magique se profile dans l'œuvre aquinienne et la dramaturgie transposée dans l'œuvre romanesque est démiurgie.

Soulignons l'importance accordée au vocabulaire supranaturel dès les premières ouvres, le recours réitéré au médecin, sorcier moderne, mais surtout au pharmacien, grand manipulateur de formules. En fait, la notion se précise: dans Prochain épisode, il n'est encore question que de sorcellerie et de magie, pouvoirs dits primitifs. Dans Trou de mémoire, on s'achemine vers 
l'alchimie, notion déjà plus sophistiquée et amplement développée dans l'Antiphonaire. On ne peut non plus s'empêcher de constater les nombreux emplois de l'adjectif "noir» ou de ses équivalents comme l'évocation de l'œuvre au noir. Car, chez Aquin, l'écriture elle-même est magique: le narrateur et $\mathrm{H}$. de Heutz se charment mutuellement par le langage (P.E., 62 et 82). C'est pourquoi le mystère de l'écriture procède du mystère alchimique mais plus encore de la mystagogie dont nous retrouvons tout le lexique: magie, occultisme, religion.

L'écrivain sera alors agent et victime: Je suis ensorcelé par la parole que je secrète (T. M., 108). De sorte que dans cette cérémonie, le narrateur sera le seul célébrant mais aussi le seul initié et le seul fidèle. On peut parler de liturgie autant que de christologie (T. M., 19, 24; etc.). Si bien que l'écriture d'Aquin est une tentative constante "d'euphémisme théologal" (cf. je suis, $\dot{a}$ moi seul, une vivante et interminable pentecôte, T.M., 26). Dans un réseau inextricable d'interférences, la lexicologie, les genres littéraires et les perspectives tentent de traduire chez Aquin le mal d'être sur l'écorce impénétrable du réel (N.N., 187) qui procède d'un au-delà irréductible.

C'est pourquoi ces formes de distanciation que sont l'humour et l'ironie s'effacent peu à peu et laissent la place à ce que l'on peut appeler le dernier texte d'Aquin: son suicide. Et l'œuvre nous apparaît comme une messe dont il serait l'offrande. Les allusions abondent qui associent Aquin à un nouveau messie souffrant sa passion. Et l'auteur, conscient de cet excès marginal, cherche à s'en distancer en le tournant d'abord en dérision: les péripéties hostiaques (T. M., 19), peuvent être prises au sens blasphématoire strict. Mais ce serait oublier la polysémie du texte soulignée trois lignes plus bas.

Pour un chrétien, le sacrifice de la messe est réalité car il est présence réelle, toujours énigmatique dans sa simplicité, sans cesse réinventé. Communion parfaite et totale avec Dieu. L'écriture va de même chez Aquin. Nous avons déjà souligné chez cet auteur la fonction démiurgique liée à la forme dramaturgique de l'écriture. Seulement, Aquin va encore plus loin puisqu'il tend à être Dieu et pas seulement à l'imiter, reprenant en cela le vocabulaire consacré à l'Eucharistie: Il n'y a plus aucun doute, ni la moindre raison d'en douter; je suis présent d'une présence réelle... (T.M., 20) ${ }^{12}$. Les mêmes signifiés se retrouveront dans Obombre dépouillés de toute ironic: le tourment se nomme. Dans ce texte, Aquin se révèle un écrivain en détresse face à l'ampleur du projet d'écrire. C'est un écrivain désabusé qui tente encore de croire. Dans Trou de mémoire l'impossibilité d'écrire n'avait pas la même acuité: Aquin évaluait bien l'importance du défi mais voulait se croire capable de le relever:

...j'incarne une image archétypale de pharmacien (...) envoûté par la mort, la surexistence ou la façon de passer de l'un à l'autre le plus élégamment possible.

Peu à peu, la puissance du mot devient hallucinatoire, non pas au sens métaphorique, mais bien comme un réel perçu dans son incessante genèse: le quotidien est sans cesse transcendé par la magie du verbe qui restitue une 
réalité (N.N., 147), à la fois connue et autre. S'affirmant d'abord comme politique ${ }^{13}$, puis esthétique, l'entreprise littéraire se révèle de plus en plus théologale.

La littérature cherche son essence dans un au-delà sacré, ultime et unique Réalité. C'est là un point fondamental de l'écriture aquinienne qui ne peut se comprendre, pensons-nous, sans l'idée d'une volonté de substitution à Dieu. Avec un titre tel qu'Obombre, on peut se demander si Aquin n'a pas voulu transposer dans son œuvre cette incarnation de la lumière divine: tout comme la lumière divine, la littérature éclairerait le monde tel qu'il est tout en l'informant de l'existence d'un autre monde. Notre monde ne serait que reflets, multiples et pâles, d'un autre monde, réel, au mystère impénétrable. Dans ce monde de plénitude assimilable à la "vraie Vie» du chrétien, tous les sens s'exerceraient ensemble, d'où les métamorphoses et les trompe-l'œil de l'œuvre littéraire. L'écrivain aurait le privilège d'être de ce monde tout en se laissant pénétrer par les arcanes de l'outre-monde (mort/surexistence) et en projetant la "lumière littéraire " ${ }^{14}$. En deça et au-delà, l'écrivain aurait le pouvoir d'être admis devant et dans l'essence même de l'image comme on dit du saint qu'il est à la fois devant Dieuls et en Lui sans pourtant Le "voir". Désormais, le temps conventionnel éclate: j'immane écrit Aquin et il peut [s]'installer en pleine transcendance (T. M., 25).

C'est là, dans cette zone incernable (et pas nécessairement religieuse) que se situerait le sacré. L'écrivain, se chargeant alors de le révéler en littérature qui, de ce fait, ne pourrait être que sacrée (ou sacrilège). Le sacrilège, imposture du sacré (T.M., 104) devient imposture littéraire (T. M., 105). Dans cette quête inlassable de l'Unité qui toujours le fuit, l'écriture, conçue comme une thérapie devient un envoûtement dont l'auteur ne peut se défaire, une liturgie qui l'écartèle encore plus.

Aquin clame ses affinités avec la tragédie grecque antique et le drame shakespearien où les dieux condamnent l'homme à la dérision et au néant: il pratique le culte de sa propre mort, met en scène et assiste au spectacle de sa propre néantisation (cf. Fortinbras). L'écriture romanesque d'Aquin rejoint le questionnement de la littérature dite "moderne» et tente de relever le même défi, à la fois ontologique et épistémologique. À ce titre, il s'agit d'une entreprise comparable à l'entreprise théologale: citer Dieu (i.e. la littérature), parler de Lui, avec Lui. Et peu à peu, l'entreprise théologale couvre entièrement l'objectif littéraire qu'elle propulsait et qui cherche pourtant à lui résister ou à la nier. On ne peut concevoir l'œuvre aquinienne, pensons-nous, sans la notion de sacré/sacrilège. Elle se fonde sur une fonction démiurgique ( Dieu dit...et cela fut), en tentant d'éclipser Dieu et en ne parlant que de Lui, autrement dit en se donnant simultanément comme sa propre fin et son propre commencement, dedans et dehors. Or, cette entreprise relève du mythe comme le prouve la littérature existante, mais un mythe nécessaire. Les armes sont inégales: Dieu seul est devant et autour ${ }^{15}$. Mais, tout en étant acculé à une défaite certaine, il s'agit de résister et en cela d'être pareil au héros nordique. Ce qui compte, seul, pour chercher à rétablir cette "grandeur perdue» est de poursuivre: 

... la pénible démarche de l'écrivain 'qui cherche à reconstituer le
roman qu'il a perdu et continue de perdre tout en l'inventant.

À ce prix est la vie mais aussi la mort.

1. Nous incluons les Rédempteurs dans l'œuvre romanesque d'Aquin. Lui-même le qualifie de «roman» dans une lettre adressée le 24-07-1952 à son ami Louis-Georges Carrier.

2. Je ne cherche pas quoi dire, mais comment le dire, P. F., 19.

3. ... il y a une espèce de présence dramatique qui est devenue lancinante et qui m'a obsédé surtout jusque dans Neige Noire et d'une façon qui m'a beaucoup surpris (Q.L.2, 135).

4. La langue d'Aquin concourt pleinement à ce projet perspectiviste globalisant: profusion verbale, multiplicité des niveaux de langage, jeux de mots, bilinguisme, néologismes, rhétorique, etc. L'entité «langue» se manifeste par sa diversité. Il aurait été intéressant aussi de mettre en parallèle théâtre et langue aquinienne: chausse-trappes et jeux de mots, décors et niveaux de langage, etc. Mais cela nous aurait trop éloignée de notre propos.

5. Soulignons que Obombre vient du verbe latin "obumbro" réservé à la conception divine: et virtus Altissimi obumbrabit tibi annonce l'Ange à Marie. Nulle part ailleurs, en linguistique, on ne retrouve ce verbe dans un autre sens; et faut-il rappeler l'érudition d'Aquin formé chez les Pères?

6. P. Smart: Hubert Aquin, agent double, 98.

7. Nous voyons aussi dans Sylvie une image inversée d'Eva: d'abord comparée à une Vierge (N.N., 44-45), elle devient peu à peu l'expression d'une dégénérescence plutôt que d'une régénération (mutilation de Nicolas, inceste, muscles du chagrin).

8. Nous soulignons.

9. Blocs erratiques, 216 .

10. Cité par Gilles de la Fontaine dans Hubert Aquin et le Québec. 16. Andrée Yanacopoulo témoignait en 1977: Ce jour-là [jour du suicide ], notre trinité en était à la dernière Cène. Québec français. mai 1977, p. 14. Voir aussi Gordon Sheppard: Signé Hubert Aquin, Montréal, Boréal-Express, 1985.

11. Édith Hamilton, la Mythologie, 375 .

12. Aquin a admiré très tôt le triomphe eucharistique de James Joyce et les innombrables épiphanies du célèbre romancier irlandais (P. F., 24).

13. Un fait politique ressemble à un succédané de religion; Blocs erratiques, 53 .

14. ...je frémis dans mon immanence et j'exécute une danse de possession à l'intérieur d'un cercle prédit (P.E., 48).

15. Dieu serait l'image parfaite qui ne renvoie qu'à elle-même. Parfaite en ce qu'elle contient tous les contraires et parce qu'elle n'a pas besoin de signifier pour être. Mais plutôt, parce qu'elle est, elle signifie.

16. Blocs erratiques, 272.

\section{ABRÉVIATIONS}

A. : l'Antiphonaire; Montréal, Le Cercle du Livre de France, 1969, 256 p.

N.N. : Neige noire; Montréal, Éditions La Presse, 1974. 254 p.

O. : Obombre; premières pages d'un dernier roman inachevé publiées dans la revue Liberté, no 135, mai-juin 1981, p. 16 à 21 .

P.E. : Prochain épisode; Montréal, Le Cercle du Livre de France, 1977, 174 p. 
P. F. : Point de fuite; Montréal, le Cercle du Livre de France, 1971, 161 p.

Q.L.2: revue Québec Littéraire, no 2. 1976; collectif spécial sur Hubert Aquin; Montréal, Éditions Guérin, $157 \mathrm{p}$.

R : les Rédempteurs; Montréal, «Les Écrits du Canada français», p. 45 à 114.

T. M. : Trou de mémoire; Montréal, Le Cercle du Livre de France, 1968, 204 p.

\section{BIBLIOGRAPHIE}

Blocs erratiques; recueil de textes rassemblés et présentés par René LAPIERRE: Montréal, Éditions Quinze, 1977, $284 \mathrm{p}$.

DE LA FONTAINE, Gilles: Hubert Aquin et le Québec, Montréal, Éditions Parti-Pris, 1977. $157 \mathrm{p}$.

HAMILTON, Édith; la Mythologie, Verviers, Marabout Université, 1940, 415 pages.

LAPIER RE, René: les Masques du récit, lecture de Prochain Épisode de Hubert Aquin, Montréal, Ếditions Hurtubise HMH, 1980, $136 \mathrm{p}$.

LAPIERRE. René: l'Imaginaire captif, Montréal, Éditions Quinze, 1981, 186 p.

MACCHABEE-IQBAL, Françoise: Hubert Aquin, romancier, Québec, Presses de l'Université Laval, 1978, 288 p.

PURDY, Anthony George, Analyse du roman Prochain Épisode: une dialectique d'illusions, Ottawa, 1982.

SMART, Patricia: Hubert Aquin, agent double. La dialectique de l'art et du pays dans Prochain Épisode et Trou de Mémoire. Montréal, P. U.M., 1973, 138 p.

Revue VOIX ET IMAGES. Vol. I, no 1, septembre 1975; dossier collectif: «Hubert Aquin et le jeu de l'écriture".

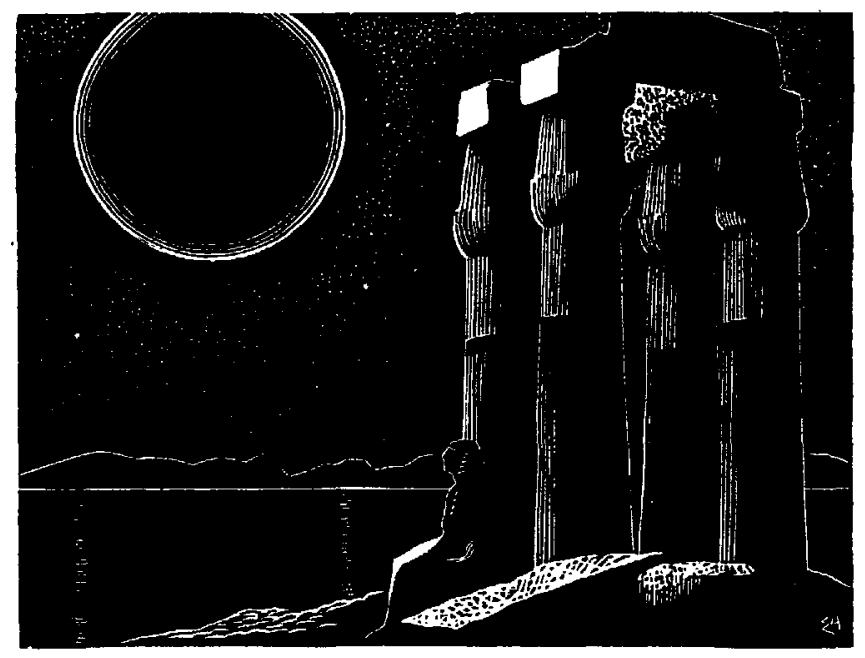

